\title{
Correspondence Analysis of Longitudinal Data
}

\author{
Peter G.M. Van Der Heidden \\ Volume 2, pp. 1230-1234 \\ In \\ Encyclopedia of Biostatistics \\ Second Edition \\ (ISBN 0-470-84907-X) \\ Edited by
}

Peter Armitage \& Theodore Colton

(c) John Wiley \& Sons, Ltd, Chichester, 2005 


\section{Correspondence Analysis of Longitudinal Data}

Correspondence analysis is an exploratory tool for the analysis of association(s) between categorical variables. Usually, the results are displayed in a graphical way.

There are many interpretations of correspondence analysis. Here we make use of two of them. A first interpretation is that the observed categorical data are collected in a matrix, and correspondence analysis approximates this matrix by a matrix of lower rank [12]. This lower rank approximation of, say, rank $M+1$ is then displayed graphically in a $M$-dimensional representation in which each row and each column of the matrix is displayed as a point. The difference in rank between the rank $M+1$ matrix and the rank $M$ representation is matrix of rank 1 , and this matrix is the product of the marginal counts of the matrix, that is most often considered uninteresting. This brings us to the second interpretation, that is, that when the two-way matrix is a contingency table, correspondence analysis decomposes the departure from a matrix where the row and column variables are independent $[8,9]$. Thus, correspondence analysis is a tool for residual analysis. This interpretation holds because for a contingency table estimates under the independence model are obtained from a product of the margins of the table (divided by the total sample size).

Longitudinal data are data where observations (e.g. individuals) are measured at least twice using the same variables. We consider here only categorical (i.e. nominal or ordinal) variables, as only this kind of variables is analyzed in standard applications of correspondence analysis [7].

\section{Two Time Points}

When there is one categorical variable measured at two time points, a so-called transition matrix can be constructed [1]. In this transition matrix, the row variable is the categorical variable measured at time 1 , and the column variable is the categorical variable at time 2. The aim of a correspondence analysis of a transition matrix is to get an insight into the transitions from time 1 to time 2. Different questions about these transitions exist, and these lead to different form of correspondence analysis.

We index the levels of the row variable (time 1) with $i,(i=1, \ldots, I)$ and the levels of the column variable (time 2$)$ with $j,(j=1, \ldots, J)$. We denote relative frequencies by $p_{i j}$, probabilities by $\pi_{i j}$, and estimates of probabilities by $\hat{\pi}_{i j}$. Marginal elements are found by replacing the index by "+", for example, row marginal elements of the matrix with relative frequencies are $p_{i+}$ and column marginal elements are $p_{+j}$.

A first analysis would be a standard correspondence analysis of the contingency table with elements $p_{i j}$. The interpretation discussed above shows that the resulting graphic display can be interpreted as showing a decomposition of the residuals from the independence model, that is, $\hat{\pi}_{i j}=p_{i+} p_{+j}$ [7-9].

A problem with this standard analysis is that often interest goes out to the off-diagonal elements (i.e. the cells for which $i \neq j$ ) in the contingency table, as these represent the individuals that change. In a standard correspondence analysis, the view on these cells might be blurred by the diagonal cells, especially, when $p_{i j} \gg p_{i+} p_{+j}$ (which is the case when many individuals remain in the same level of the categorical variable from time point 1 to 2). A solution to this problem is not to study the residuals from the independence model, but from the so-called quasi-independence model, defined here as $\pi_{i i}=p_{i i}$ for $i=j$ and $\pi_{i i}=\alpha_{i} \beta_{j}$ for $i \neq j$ [1]. It is possible to adjust correspondence analysis so that residuals from quasi-independence are decomposed. This can be done in two ways: by adjusting the computer program or by changing the input data. The last option seems most simple, and the way to do it is as follows: the diagonal elements $p_{i i}$ have to be replaced by elements for which independence holds. This can be accomplished by filling in elements $p_{i+} p_{+i}$ for the diagonal. By doing this, the margins of the new table have changed so that the elements on the diagonal are not independent, and therefore, using the new margins, again elements $p_{i+} p_{+i}$ have to be filled in. After a few iterations, these elements have stabilized, and a correspondence analysis of the resulting table can be interpreted as a decomposition of quasi-independence $[7,8,11]$.

This approach can be extended further by adjusting correspondence analysis so that it can decompose residuals from the symmetry model or from the quasi-symmetry model [7, 8]. Another development 
is to use statistical models instead of the exploratory approach described here. There are also close connections between correspondence analysis and latent class analysis [12].

We give a small example to illustrate an analysis of the departure from independence. Space limitations withhold us from a detailed interpretation, and for interpretation principles, we refer to correspondence analysis. The data are 5 import car types out of 16 car types published in [8]: subcompacts (subi), small specialties (smai), compacts (comi), midsize (midi), and luxury (luxi). In the rows of Table 1, we find the cars disposed of, and in the columns the new cars. Notice the dominant observed frequencies on the diagonal. These values dominate the first dimensions of a correspondence analysis (see Figure 1), especially, the diagonal luxi-cell compared with the rest. In a second analysis, we decompose the residuals from quasi-independence. Such an analysis can be

Table 11979 car changing data

\begin{tabular}{lrrrrrr}
\hline & \multicolumn{1}{c}{ subi } & smai & comi & midi & luxi & Total \\
\hline subi & 25986 & 5400 & 2257 & 1307 & 288 & 35238 \\
smai & 3622 & 5249 & 738 & 1070 & 459 & 11138 \\
comi & 6981 & 1023 & 1536 & 1005 & 127 & 10672 \\
midi & 2844 & 772 & 565 & 3059 & 595 & 7835 \\
luxi & 997 & 341 & 176 & 589 & 3124 & 5227 \\
Total & 40430 & 12785 & 5272 & 7030 & 4593 & 70110 \\
\hline
\end{tabular}

Rows denote cars disposed, columns denote new cars. Abbreviations are in the text. accomplished by filling in "independent" values for the diagonal. These values are 12 790, 1381, 1033, 503 and 71 . The interpretation of this correspondence analysis uses the same principles as for standard correspondence analysis of the table with the adjusted margins. For the margins, the residuals are zero, and therefore, the graph only shows car type changes. The car order for cars disposed off is luxi, midi, comi, subi, and smai, but for new cars it is luxi, midi, smai, comi, and subi (see Figure 2). Notice, for example, the different position of smai. It is due to asymmetries in the data that become visible now that the dominance of the diagonal elements has been suppressed. For example, when people dispose of a smai, they buy a luxi very often (relative to the margins of the adjusted table, i.e. observed 459 but predicted by margins 239) but the reverse does not hold (observed 341 but predicted by margins 413 ).

\section{More than Two Time Points}

When there is one categorical variable measured at more than two time points, it is usual to code the response profiles into a so-called superindicator matrix (see Correspondence Analysis). Correspondence analysis of a superindicator matrix is also known as multiple correspondence analysis. A superindicator matrix has $N$ individuals in the rows and the categories for each of the time points in the columns. This correspondence analysis has the aim to

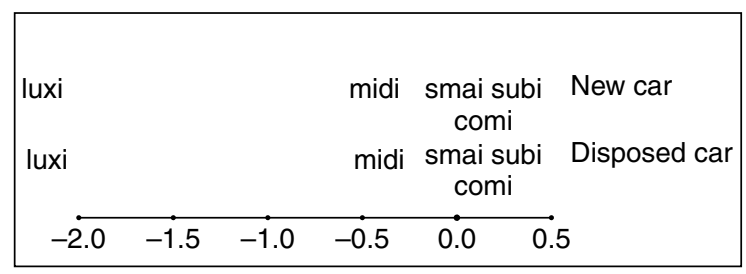

Figure 1 Ordinary CA of car changing data

\begin{tabular}{|ccccc|}
\hline luxi & midi & & & \\
& & $\begin{array}{l}\text { comi smai } \\
\text { luxi } \\
\text { midi }\end{array}$ & smai & New car \\
comi subi & Disposed car \\
-2.0 & -1.5 & -1.0 & -0.5 & $0.0 \quad 0.5$ \\
\hline
\end{tabular}

Figure 2 Generalized CA decomposing residuals from quasi-symmetry 
get insight into the transitions between all time points simultaneously. The analysis also yields quantifications for the individuals, and the quantifications for an individual can be considered as summaries of the response profile of this individual that can be used, but it can also be used to obtain a classification of the response profiles of the individuals $[2-7,10]$.

As an example, we give a superindicator matrix of one dichotomous variable measured at three time points for $N=101$ individuals (see Table 2). (In many computer programmes, the column vector with frequencies cannot be specified, but instead a matrix with 101 rows will serve as the data input file.) The matrix can be made larger in a straightforward way when the number of categories is larger than two, when there are more time points, or when there are more individuals. A correspondence analysis of this matrix will yield a three-dimensional display with 101 points, one for each individual, and a graphical display with 8 points, one for each category at each time point. Without going into technical details (see $[5,10])$, individuals with similar profiles will be close together, categories that are often used by the same individuals will be close together, and, when we overlay the two graphs, individuals will be close to the categories that they use. It is also important to notice that, since correspondence analysis displays the departure from the row and from the column margin of a table, it follows that correspondence analysis will not show the trend in "a" and "b" over the three time points. This trend can be studied from the counts in the $3 \times 2$ table of time points by categories [7, 10].

Another way to interpret this analysis is when we realize that a correspondence analysis of the

Table 2 A small example of a categorical data matrix (panel A) and its superindicator matrix (panel B)

\begin{tabular}{|c|c|c|c|c|}
\hline \multirow{2}{*}{$\begin{array}{l}\text { Panel A } \\
\mathrm{t} \\
123\end{array}$} & \multirow[b]{2}{*}{ Freq } & \multicolumn{3}{|c|}{ Panel B } \\
\hline & & $\begin{array}{c}t_{1} \\
\mathrm{a} \mathrm{b}\end{array}$ & $\begin{array}{c}t_{2} \\
\mathrm{a} \mathrm{b}\end{array}$ & $\begin{array}{c}t_{3} \\
\mathrm{ab}\end{array}$ \\
\hline $\mathrm{a}$ a a & 40 & 10 & 10 & 10 \\
\hline $\mathrm{a} a \mathrm{~b}$ & 16 & 10 & 10 & 01 \\
\hline $\mathrm{a} b \mathrm{a}$ & 4 & 10 & 01 & 10 \\
\hline $\mathrm{a} b \mathrm{~b}$ & 12 & 10 & 01 & 01 \\
\hline $\mathrm{b}$ a a & 8 & 01 & 10 & 10 \\
\hline $\mathrm{b}$ a b & 3 & 01 & 10 & 01 \\
\hline $\mathrm{b} b \mathrm{a}$ & 6 & 01 & 01 & 10 \\
\hline $\mathrm{b} b \mathrm{~b}$ & 12 & 01 & 01 & 01 \\
\hline
\end{tabular}

Table 3 The Burt matrix for the example in Table 2

\begin{tabular}{|c|c|c|c|c|c|c|c|}
\hline & & \multicolumn{2}{|c|}{$t_{1}$} & \multicolumn{2}{|c|}{$t_{2}$} & \multicolumn{2}{|c|}{$t_{3}$} \\
\hline & & $\mathrm{a}$ & b & $\mathrm{a}$ & b & $\mathrm{a}$ & $b$ \\
\hline \multirow[t]{2}{*}{$t_{1}$} & $\mathrm{a}$ & 72 & 0 & 56 & 16 & 44 & 28 \\
\hline & b & 0 & 29 & 11 & 18 & 14 & 15 \\
\hline \multirow[t]{2}{*}{$t_{2}$} & $\mathrm{a}$ & 56 & 11 & 67 & 0 & 48 & 19 \\
\hline & b & 16 & 18 & 0 & 34 & 10 & 24 \\
\hline \multirow[t]{2}{*}{$t_{3}$} & $\mathrm{a}$ & 44 & 14 & 48 & 10 & 58 & 0 \\
\hline & b & 28 & 15 & 19 & 24 & 0 & 43 \\
\hline
\end{tabular}

superindicator matrix, say $\boldsymbol{G}$, is mathematically related to a correspondence analysis of the so-called Burt matrix $\boldsymbol{G}^{\prime} \boldsymbol{G}$ (see Correspondence Analysis). The Burt matrix for this example is shown in Table 3. This matrix is a concatenation of a twoway contingency table for each pair of time points, and diagonal matrices with marginal frequencies. This shows that the solution of correspondence analysis only uses two-way interactions, and ignores higher-way interactions. Thus, a Burt matrix contains sufficient information for a nonstationary Markov chain (the table of time points 1 and 3 is the matrix product of the tables of time points 1 and 2, and 2 and 3) $[7,10]$.

Examples of such analyses can be found in [2-4, $7,10]$. If the number of individuals is not very large, the estimates for the category points will be unstable. More stability is obtained by constraining category points of adjacent time points to be the same. Such a solution can be obtained by adding up the indicator matrices of the adjacent time points [6]. This is also the way to go when the data to be analyzed are event history data, where the observations are in continuous time, or career data. Examples of unconstrained and constrained analyses are in $[3-7,10]$.

\section{References}

[1] Bishop, Y.M.M., Fienberg, S.E. \& Holland, P.W. (1975). Discrete multivariate analysis. M.I.T.Press, Cambridge.

[2] de Leeuw, J., van der Heijden, P.G.M. \& Kreft, I. (1985). Homogeneity analysis of event history data, Methods of Operations Research 50, 299-316.

[3] Deville, J.-C. \& Saporta, G. (1983). Correspondence analysis, with an extension towards nominal time series, Journal of econometrics 22, 169-189.

[4] Martens, B. (1994). Analyzing event history data by cluster analysis and multiple correspondence analysis: an example using data about work and occupations of scientists and engineers, in Correspondence analysis in 
the social sciences, M. Greenacre \& J. Blasius, eds. Academic Press, London, 233-251.

[5] Saporta, G. (1985). Data analysis for numerical and categorical individual time-series, Applied stochastic models and data analysis 1, 109-119.

[6] van Buuren, S. \& de Leeuw, J. (1992). Equality constraints in multiple correspondence analysis, Multivariate behavioral research 27, 567-583.

[7] van der Heijden, P.G.M. (1987). Correspondence analysis of longitudinal categorical data. D.S.W.O.-Press, Leiden.

[8] van der Heijden, P.G.M., de Falguerolles, A. \& de Leeuw, J. (1989). A combined approach to contingency table analysis using correspondence analysis and loglinear analysis, Applied Statistics 38, 249-292.

[9] van der Heijden, P.G.M. \& de Leeuw, J. (1985). Correspondence analysis used complementary to loglinear analysis, Psychometrika 50, 429-447.
[10] van der Heijden, P.G.M. \& de Leeuw, J. (1989). Correspondence analysis, with special attention to the analysis of panel data and event history data, in Sociological Methodology 1989, C.C. Clogg, ed. Basil Blackwell, Oxford, 43-87.

[11] van der Heijden, P.G.M., de Vries, H. \& van Hooff, J.A.R.A.M. (1990). Correspondence analysis of transition matrices, with special attention to missing entries and asymmetry, Animal Behaviour 40, 49-64.

[12] van der Heijden, P.G.M., Gilula, Z. \& van der Ark, L.A. (1999). An extended study into the relationships between correspondence analysis and latent class analysis, in Sociological Methodology 1999, M. Sobel \& M. Becker eds. Blackwell, Cambridge, pp 147-186.

Peter G.M. Van Der HeIJden 